\title{
P Cygni in a short S Doradus phase. Spectroscopic and photometric evidences
}

\author{
N. Markova ${ }^{1}$, N. Morrison ${ }^{2}$, I. Kolka ${ }^{3}$, and H. Markov ${ }^{1}$ \\ 1 Institute of Astronomy and Isaac Newton Institute of Chile Bulgarian Branch, Bulgarian National Astronomical \\ Observatory, PO Box 136, 4700 Smoljan, Bulgaria \\ 2 Ritter Observatory, The University of Toledo, Toledo, OH 43606, USA \\ 3 Tartu Observatory, Tõravere 61602, Estonia
}

Received 22 March 2001 / Accepted 02 May 2001

\begin{abstract}
We studied the long-term spectral and photometric behaviour of P Cygni over the 13.8 year interval from March 1985 to January 1999. The $U B V$ photometry reveals a slow ( 7.4-year), low-amplitude $(\sim 0.1 \mathrm{mag})$ variation in $V$ in which the star becomes redder when it brightens and vice versa. It underwent a possible maximum in the winter of 1985 (between JD 2446000 and JD 2446200 ), a minimum in the winter of 1989 (between JD 2447500 and JD 2447 700), a maximum in the fall of 1992 (between JD 2448800 and JD 2449 000), and a minimum in the spring of 1996 (between JD 2450100 and JD 2450300 ) The properties of this variation are typical for weak-active S Dor variables in "short S Dor phase" (van Genderen 2001). Simultaneous spectroscopic observations show changes in the $\mathrm{H} \alpha$ equivalent width (corrected for the effect of the changing continuum) in positive correlation with the 7.4-year photometric oscillation in the $V$-band brightness. This result is interpreted as an indication that in P Cygni an increase in the stellar brightness, during the 7.4-year SD phase, is likely accompanied by an increase in the mass-loss rate. In this behaviour P Cygni is similar to S Dor and R71. From simple considerations, it is concluded that the 7.4-year SD cycle is probably a combination of an increasing radius/decreasing effective temperature and an expanding pseudo-photosphere.
\end{abstract}

Key words. stars: oscillations - stars: mass loss - stars: individual: P Cygni

\section{Introduction}

Luminous Blue Variables (LBVs) form a class of very massive, unstable stars located near the Humphreys-Davidson upper luminosity limit in the Hertzprung-Russell diagram. The term "LBVs" was coined by Peter Conti in 1984 and comprises the galactic P Cygni type stars, the S Doradus variables and the Hubble-Sandage variables in M 31 and M 33. The evolutionary status of these objects is still a subject of ongoing debates. In some stellar models, the LBV phase occurs during (or shortly after) the mainsequence phase, produces an enormous mass loss, and prevents evolution to the red (Schaller et al. 1992; Meynet et al. 1994; Langer et al. 1994); in others, the LBVs are post-red-supergiant objects in a late blue phase of dynamical instability (Stothers \& Chin 1996, 2000). Some believe that two types of LBVs appear to exist: "classical" LBVs with $M_{\text {bol }}$ brighter than -9.5 mag and "low luminous" LBVs with $M_{\text {bol }}$ weaker than $-9.5 \mathrm{mag}$, both of which

Send offprint requests to: N. Markova,

e-mail: rozhen@mbox.digsys.bg share the main characteristics of the class, but may be in different evolutionary state (e.g. Humphreys \& Davidson 1994).

Van Genderen (2001) has recently published an extensive review of the 20th century scientific achievements on LBVs where 46 confirmed and candidate members of the class are listed. He also updated the criteria for membership in the class from those given by Humphreys \& Davidson (1994) introducing new terms, such as "S Dor (SD)-eruption" and "S Dor (SD)-phase". The last one is a photometric variation of up to $2.5 \mathrm{mag}$ on a time scale of years to decades and even to centuries. A crucial feature of this variability is that the star becomes redder when it brightens and vice versa. In addition, van Genderen (2001) argued that the term "S Doradus variables" should replace "LBV". A definition has been formulated by a small committee at the Workshop "P Cygni 2000: 400 years of progress" (de Groot \& Sterken 2001).

Historically, the hypergiant P Cygni is the first known LBV. The star satisfies the first two criteria for membership in the class. It has undergone at least one SD-eruption 
(17th century). It has a high luminosity, $\log L / L_{\odot}=$ 5.86 (Pauldrach \& Puls 1990), a high mass-loss rate, $\sim 2 \times 10^{-5} M_{\odot} \mathrm{yr}^{-1}$ (Scuderi et al. 1994, 1998; Markova et al. 2001), an extended atmosphere and four visible ejecta containing processed material (Barlow et al. 1994; Meaburn et al. 1996; Skinner et al. 1997; Johnson et al. 1992). Surprisingly, no indication for any SD-phases was known until Markova et al. (2001) and Markova (2001) reported suggestive evidence for a very slow variation in the stellar brightness with an amplitude of about $0.13 \mathrm{mag}$ and a duration of about 7 years. During this variation, when the star brightens, the effective temperature decreases by about $10 \%$ and the radius increases by about $7 \%$ leaving the luminosity almost constant. Simultaneous $\mathrm{H} \alpha$ spectroscopy suggests that the $V$-band variability is accompanied by changes in the properties of the wind, namely mass-loss rate and velocity field. Unfortunately, due to the time limitation of the available data sets Markova et al. were not able neither to estimate confidently the time scales, amplitudes, and recurrence of the variability, nor to elucidate the relationship between the spectral and the photometric variability.

The aims of our study are to determine the properties of the very long-term variability of $\mathrm{P}$ Cygni, to establish the character of the relationship, if any, between variations in the wind and in the photosphere, and thus to obtain some insight into the origin of the variability. For this purpose, we collected and analysed $U B V$ photometry and $\mathrm{H} \alpha$ line profiles or equivalent widths obtained over a period of 13.8 years. The observational material used is described in Sect. 2, where some problems concerning the homogeneity of our sample are discussed and resolved. The results obtained are described in Sect. 3, and the physical nature of the variability is discussed in Sect. 4.

\section{Observations and measurements}

\subsection{Photometric observations}

The $U B V$ photometric data presented here are taken from de Groot et al. (2001a,b) and Percy et al. (1996). The data cover the period JD 2446174 to JD 2451 102, which corresponds to March 1985 to October 1998. Some of them have already been analysed by Scuderi et al. (1994) and Markova et al. (2001). The distribution of the data by means of their origin is given in Table 1, which also lists the accuracy in $V$ and $B-V$.

The $\mathrm{H} \alpha$ spectroscopic observations were carried out at the National Astronomical Observatory (NAO), Bulgaria, at Ritter Observatory (RO), USA; and at the Tartu Astrophysical Observatory (TAO), Estonia. The observations at the NAO (Markova) were obtained using the coudé spectrograph of the 2-m RCC telescope. They consist of nine photographic spectra (Kodak 103aF) with resolving power $R(\equiv \lambda / \Delta \lambda)=18200$ and $7 \mathrm{H} \alpha \mathrm{CCD}$ spectra with $R=15200$ and a wavelength coverage of about $110 \AA$ taken during May-September 1990 and April-October 1998, respectively. For the latter, the detec-
Table 1. Summary of photometric observations. $N$ denotes number of observations.

\begin{tabular}{llll}
\hline Author & N & $\begin{array}{l}\text { Accuracy } \\
\text { in V, } B-V\end{array}$ & Reference \\
\hline Percy & 300 & not reported & Percy et al. 1988 \\
Zsoldos & 10 & not reported & private communication \\
CAMC & 124 & $0.05 ;--$ & Carlsberg 1985-1994 \\
APT & 220 & $0.006 ; 0.010$ & Genet et al. 1987 \\
\hline
\end{tabular}

tor was an ELECTRON ISD015A CCD with $520 \times 580$ pixels and a pixel size of $18 \times 24 \mu \mathrm{m}$. The CCD frames were uniformly reduced by means of a series of modules written in IDL by T. Valchev. The procedure is entirely standard and consists of background subtraction, cosmic ray hit removal, flat-fielding, wavelength calibration, and continuum normalisation. Pixel-to-pixel sensitivity variation is removed by division by the mean flat field for the relevant night. During the wavelength calibration, the Earth's motion with respect to the heliocentric rest frame is removed.

Observations at Ritter Observatory were carried out by members of the observing team with a fiber-fed échelle spectrograph attached to the 1-m telescope and to a liquid-nitrogen-cooled Wright Instruments Ltd. CCD camera, which incorporates an $770 \times 1152$ EEV CCD0520-0-202 sensor with pixel dimensions of $22.5 \times 22.5 \mu \mathrm{m}$. Nine échelle orders, each with a spectral range of about $70 \AA$ and $R=25800$, were included; only the $\mathrm{H} \alpha$ range is discussed here. The raw frames were reduced with Ritter Observatory's standard techniques (Mulliss 1996) under Sun/IRAF V2.10.4-p1 ${ }^{1}$. Bias subtraction, flat fielding, wavelength calibration, heliocentric correction, and continuum normalisation were carried out with standard IRAF tasks.

Observations at the TAO (Kolka) were carried out with a Cassegrain spectrograph (ASP-32) attached to $1.5-\mathrm{m}$ telescope. The spectrograph was equipped with an ST-6 CCD cameras $(242 \times 375$ pixels; observation window $90 \AA)$ from June 1994 to September 1995 and with an HPC$1(1024 \times 1024$ pixels; observation window $250 \AA)$ afterwards. In both cases the resolution is about $0.24 \AA /$ pixel. The initial CCD reduction operations (dark and bias subtraction etc.) were performed with the MIDAS package. Wavelength calibration, continuum normalisation, heliocentric correction, and $E W$ measurements were carried out by means of the local KASPEK package (for more details see Leedjärv 1998).

The distribution of the observations in time is summarised in Table 2. The fourth and fifth columns give, respectively, the spectral resolving power, $R \equiv \lambda / \Delta \lambda$, and

\footnotetext{
${ }^{1}$ IRAF is distributed by the National Optical Astronomy Observatories, which are operated by the Association of Universities for Research in Astronomy, Inc., under contract with the National Science Foundation.
} 
Table 2. Summary of $\mathrm{H} \alpha$ observations.

\begin{tabular}{lllll}
\hline Source & Time period & $\begin{array}{l}\text { Time period } \\
\text { JD 2 440 000+ }\end{array}$ & $\begin{array}{l}\text { spectral } \\
\text { resolution }\end{array}$ & $\begin{array}{l}\text { observing } \\
\text { nights }\end{array}$ \\
\hline NAO (Bulgaria) & May - Sep. 90 & 8017 to 8137 & 18200 & 9 \\
NAO & Apr. - Oct. 98 & 10915 to 11095 & 15200 & 7 \\
TAO (Estonia) & June 94 - Jan. 97 & 9512 to 10264 & 16000 & 30 \\
RO (USA) & June 94 - Oct. 98 & 9512 to 11110 & 25800 & 39 \\
\hline Taylor et al. 1991 & May 89 - Sep. 90 & 7668 to 8159 & 193 & 32 \\
Scuderi et al. 1994 & July $88-$ Aug. 93 & 7348 to 9227 & 7000 & 139 \\
Markova et al. 2001 & June 90 - June 94 & 8044 to 9529 & 12000 to $2 \times 10^{4}$ & 128 \\
Pollmann 1999 & Sep. $94-$ Jan. 99 & 9600 to 11300 & 2000 & \\
\hline
\end{tabular}

the number of the obtained spectra (one spectrum per night).

\subsubsection{Published $\mathrm{H} \alpha$ data}

In addition to the original $\alpha$ equivalent width $(E W)$ determinations, we used published data (Scuderi et al. 1994; Taylor et al. 1991; Markova et al. 2001; Pollmann 1999).

The $\mathrm{H} \alpha$ observations used by Scuderi et al. (1994) were obtained with an échelle spectrograph and a CCD detector attached to the 91-cm Cassegrain telescope of the Catania Astrophysical Observatory. The resolving power was 7000 with a coverage of $450 \AA$. A signal-to noise ratio of about 50 in the continuum was achieved. The $E W$ estimates include the contribution of both the line core and the emission wings. A relative error of about 4 percent in $E W$ was estimated. The dataset published by Scuderi et al. (1994) is here enlarged with five new $E W$ determinations based on observations carried out at the CAO between 19 and 27 August 1993.

The data published by Taylor et al. (1991) come from low-resolution (34 $\AA$ ) spectra obtained at the Pine Bluff Observatory, which were originally designed for polarisation measurements. The $E W$ estimates include only the contribution of the line core. The spectral flux, expressed in continuum units, has been integrated within $60 \AA$ around $\mathrm{H} \alpha(6540 \leq \lambda \leq 6600 \AA)$. No information about the accuracy of the measurements is given.

The $\mathrm{H} \alpha E W$ determinations published by Markova et al. (2001) are based on CCD frames obtained at the NAO $(R \sim 20000$, spectral coverage $110 \AA)$ as well as on échelle spectra $(R \sim 12000)$ published by Stahl et al. (1994). The level of the continuum was determined by linear interpolation between the average values of the stellar counts in two bands situated at about $6510 \AA$ and at about $6617 \AA$. The line flux was than integrated over these limits. The accuracy of the determinations is $\pm 1 \AA$ or less.

The EW estimates published by Pollmann (1999) cover the period from 1994 to 1999. They originate from amateur observations made with a combination of a 100- mm Maksutov-type reflector $(f=1000 \mathrm{~mm})$, an objective prism (refracting angle of $30^{\circ}$ ) and a CCD camera (FT800P, $386 \times 290$ pixels). A $S / N$ ration of 200 was reached. The resolving power is 2000 . The equivalent width of $\mathrm{H} \alpha$ is determined by spectral flux integration within an interval of $140 \AA$, namely from 6490 to $6630 \AA$. The accuracy of the determinations is 5 percent (private communication). The resulting estimates are shown as a function of time in Fig. 2 (lower panel).

Information concerning the collected $\mathrm{H} \alpha E W$ measurements and the relevant observations is summarised in Tables 2 and 3. The total time coverage of the sample is 10 years and 6 months, from July 1988 to Jan 1999.

\subsection{Equivalent width determinations}

An important problem for our equivalent width determinations is mutual consistency among the data sets in terms of continuum placement and integration limits. The $\mathrm{H} \alpha$ $E W$ in $\mathrm{P}$ Cygni was estimated through integration between two continuum points on either side of the line. Because this line has strong emission wings extending to $\pm 32.8 \AA$ (about $\pm 1500 \mathrm{~km} \mathrm{~s}^{-1}$ ) from line centre, to obtain reliable $E W$ estimates requires that the two continuum bands be chosen close to but outside the wavelength region 6529 to $6595 \AA$. When the spectral range of the observations is not sufficient to include the true continuum on either side of $\mathrm{H} \alpha-$ as is the case with, e.g., the Ritter observations - the $E W$ s are underestimated and should be corrected for undetected line flux.

Another problem is blending effects that may depend on time, on spectral resolution, and on the geographic location of the observatory. No correction for the contribution of water vapour lines, C II doublet, or N II forbidden lines was made. However, we estimated that in most cases the total effect of blending did not exceed one percent of the $\mathrm{H} \alpha E W$. In the extreme case of Ritter Observatory, which is located essentially at sea level, the contribution of water absorption to the $\mathrm{H} \alpha$ equivalent width amounts to less than $2 \%$. 
Table 3. H $\alpha$ equivalent width measurements.

\begin{tabular}{lllll}
\hline Source & $\begin{array}{l}\text { line-flux } \\
\text { integration limits }\end{array}$ & $\begin{array}{l}\text { accuracy of } \\
\text { the measurements } \\
\text { (in percent) }\end{array}$ & $\kappa / \sigma\left(W_{\text {corr }}\right)$ & $\begin{array}{l}\text { contribution of } \\
\text { telluric lines } \\
\text { (in } \AA \text { ) }\end{array}$ \\
\hline NAO (Bulgaria) & $\begin{array}{l}6510 \text { to } 6617 \\
6519 \text { to } 6608\end{array}$ & 3 & $1.00 /--$ & $\leq 0.2$ \\
Tartu Obs. (Estonia) & & $1.01 /--$ & $\leq 1.0$ \\
Tartu Obs. after & 6510 to 6617 & 2 & $1.00 /--$ & $\leq 1.0$ \\
Sept. 1995 & 6531.5 to 6593.5 & 3 & $1.096 / 3.5 \%(4)$ & $\leq 1.2$ \\
Ritter Obs. (USA) & 6540 to 6600 & not reported & $1.25 / 5 \%(3)$ & not reported \\
\hline Taylor et al. (1991) & 6510 to 6617 & 4 & $1.00 /--$ & $\leq 0.4$ \\
Scuderi et al. (1994) & 6510 to 6617 & $<2$ & $1.00 /--$ & $\leq 0.4$ \\
Markova et al. (2001) & 6490 to 6630 & 5 & $--/--$ & not reported \\
Pollmann (1999) & & & &
\end{tabular}

\subsection{Correction for undetected $H \alpha$ line-flux}

The $E W$ corrected for undetected line-flux, $W_{\text {corr }}$, was derived as follows. The $\mathrm{H} \alpha$ profiles studied by Markova et al. (2001) were averaged to obtain a mean profile. The equivalent width found by integration between arbitrary wavelength limits, $a$ and $b$, is called $W_{a, b}$. The correction coefficient for a given dataset, $\kappa$, was derived as $\kappa=W_{6510,6617} / W_{a, b}$, where $a$ and $b$ are the integration limits relevant to the dataset in question $(a \geq 6510 \AA$ and $b \leq 6617 \AA)$. The value obtained for $\kappa$ was then applied to all data in the dataset to obtain $W_{\text {corr }}$. Obviously $\kappa=1.00$ for equivalent widths derived by integration between $6510 \AA$ and $6617 \AA$, e.g. the dataset originating from the NAO and the one obtained at the TAO after September 1995. Since the correction coefficient thus derived is based on the mean contribution of the wings to the total $E W$ of the line, individual values of $W_{\text {corr }}$ will differ from the real values if the line wings vary.

To evaluate the accuracy of the corrected equivalent widths, $\sigma\left(W_{\text {corr }}\right)$, we compared $W_{\text {corr }}$ from a given source with data from another source obtained in the same night(s) (if any) that include the total line flux between $6510 \AA$ and $6617 \AA$. We checked that this comparison is valid by studying the variability of the $\mathrm{H} \alpha E W$ within one night (using CCD spectra obtained at the NAO, Bulgaria) and found that its hourly variations are negligibly small. For example, the rms deviation derived by averaging over 25 spectra obtained within a period of 8 hours on 24 June 1994 amounts to $0.5 \%$ of the nightly mean. During the next night (total observing time 7 hours), the rms deviation averaged over 16 spectra was $0.7 \%$ of the $E W$. In this way, we found that the accuracy of individual values of $W_{\text {corr }}$ is better than $9 \%$.

The $\mathrm{H} \alpha E W$ determinations are summarised in Table 3, where: Col. 2 gives the integration limits; Col. 3 lists the accuracy of the measurements in percent; Col. 4 lists the correction coefficient for reduced spectral range, $\kappa$, and the standard deviation in the corrected equivalent

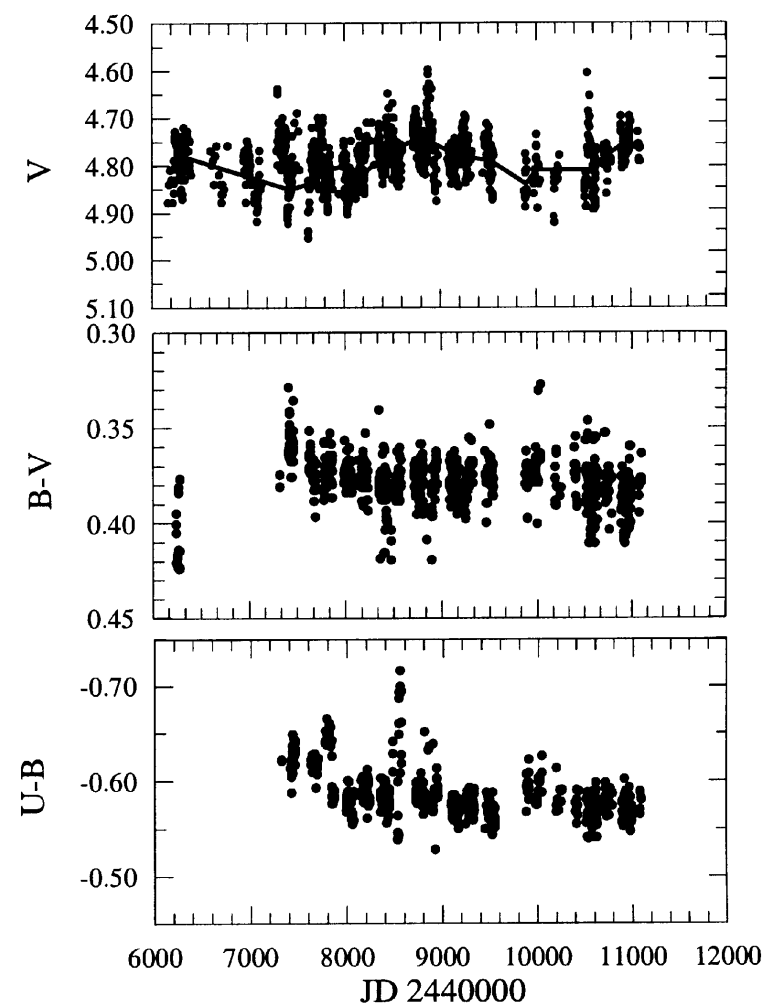

Fig. 1. Extended light and colour curves of P Cygni. Data are taken from de Groot et al. (2001a,b). The intention of the solid line, derived by averaging the data points over time windows naturally defined by major seasonal gaps in the observations, is to guide the eye and to emphasise the presence of a slow component in the photometric behaviour of the star. The amplitudes of the cyclic variation in $V, B-V$ and $U-B$ must be genuine since they exceed the accuracy of the relevant determinations (less than $0.05 \mathrm{mag}$ in $V, 0.01 \mathrm{mag}$ in $B-V$ and $0.02 \mathrm{mag}$ in $U-B)$.

widths, $\sigma\left(W_{\text {corr }}\right)$, averaged over all nights in common with the corresponding reference source (number of nights in 
parentheses); and Col. 5 gives the estimated percentage contribution of water vapour lines to the $\mathrm{H} \alpha E W$.

\section{Results}

\subsection{Photometric variability}

The light and colour curves of P Cygni over the 13.5-year period from March 1985 to October 1998 are shown in Figure 1. The solid line connects points which represent the mean values of $V$, computed over time windows naturally defined by major seasonal gaps in the observations. The intention of this line is to guide the eye and to emphasise the presence of a slow component in the photometric behaviour of the star. The data confirm the presence of the slow variation in the stellar brightness suggested by Markova et al. (2001), with the star becoming redder, according to the $B-V$ colour index, as it brightens and vice versa. Because our sample includes new data, it reveals the presence of two cycles, both incomplete. In addition, it is now evident that apart from the rapid and very strong variation between JD 2448538 and JD 2448567 the behaviour of the $U-B$ colour index tends to follow that of $B-V$. The reality of the event around JD 2448550 must be checked additionally. The variation in $V$ has a time scale of about 7.4 years and an amplitude of about $0.1 \mathrm{mag}$, typical of weak-active S Dor variables in a short SD-phase (van Genderen 2001). Thus, we conclude that P Cygni experienced a short SD-phase with a possible maximum in the winter of 1985 (between JD 2446000 and JD 2446200 ), a minimum in the winter of 1989 (between JD 2447500 and JD 2447700), a maximum in the fall of 1992 (between JD 2448800 and JD 2449000 ), and a minimum in the spring of 1996 (between JD 2450100 and JD 2450300$)$. In addition, the fact that the scatter of the data around the mean light curve exceeds the error in the individual determinations indicates real variations on a shorter time scale (de Groot et al. 2001a,b; Markova et al. 2001).

\section{2. $H \alpha$ equivalent width variability}

The $\mathrm{H} \alpha E W$ determinations, both original and collected, are shown in Fig. 2 as a function of JD. The data have been corrected as needed for undetected line flux following the procedure described in Sect. 2 . The data (upper panel) clearly indicate the presence of a slow component - we called it the Very-Long Term (VLT) component - in the $\mathrm{H} \alpha E W$ variability. The pattern of the VLT variability is illustrated by a dashed line, derived from a fourth order polynomial fit to the data. The amplitude of variation (according to the fit) is about $20 \AA$. The sharp dip in the H $\alpha E W$ curve (amplitude $\sim 30 \AA$, time scale $\sim 600^{\mathrm{d}}$ ), situated between JD 2448000 and JD 2448600 is real since it was independently recorded by Markova et al. (2001,see the detailed curve in Fig. 5) - the so-called Long-Term (LT) variation - and Taylor et al. (1991). Because of the limited duration and the low time resolution of their obser-

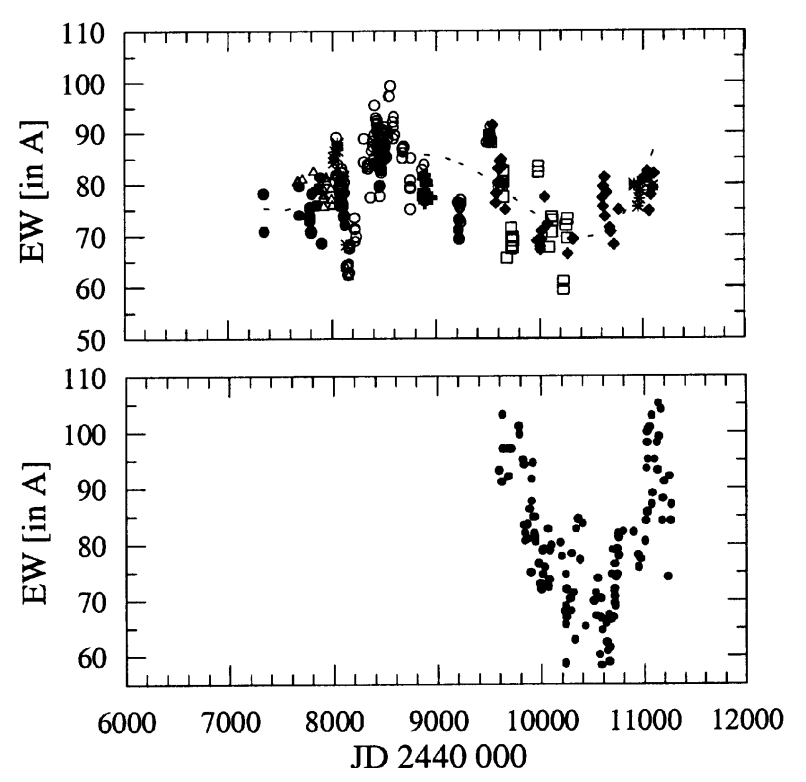

Fig. 2. $\mathrm{H} \alpha$ equivalent width variability over a period of 10.6 years (from July 1988 to January 1999) as a function of Julian date. Upper panel: data from different sources are marked with different symbols: triangles, Taylor et al. (1991); squares, Tartu Observatory; diamonds, Ritter Observatory; dots, Scuderi et al. (1994); asterisk, National Astronomical Observatory;open circles, Markova et al. (2001). The intention of the dashed line is to guide the eye and to emphasise the presence of a slow component in the $\mathrm{H} \alpha$ equivalent width variability. Lower panel: data published by Pollmann (1999).

vations Markova et al. were not able to ascertain weather this variation is a recurrent phenomenon. With the new spectral data, we are now able to say that this variation might be recurrent: the fact that the scatter of the data around the smooth VLT curve exceeds the accuracy of the $E W$ determinations by more than a factor 3 indicates real variability on a shorter time scale. The data of Pollmann (1999), shown in the lower panel of Fig. 2, seem to support this conclusion. However, there is no obvious explanation for the much larger amplitude of the VLT component that appears in the latter data, and we suspect that these data may contain an unrecognised source of systematic error.

\subsection{Correlation between stellar brightness and $\mathrm{Ha}$ equivalent width}

To learn more about the nature of the slow component in the spectral and photometric variabilities of the star and the connection between them (if any) we compared the simultaneous behaviour of the stellar brightness and the $\mathrm{H} \alpha E W$. In the top panel of Fig. 3, the solid line, a seventh-order polynomial fit to the data points, traces out the $V$-band variability. In the three panels below, the same curve (scaled in an appropriate way to fit the relevant data) is represented by a dashed line. The purpose of these curves is to guide the eye and to make the comparison easier. From the two panels in the middle of Fig. 3, it is 

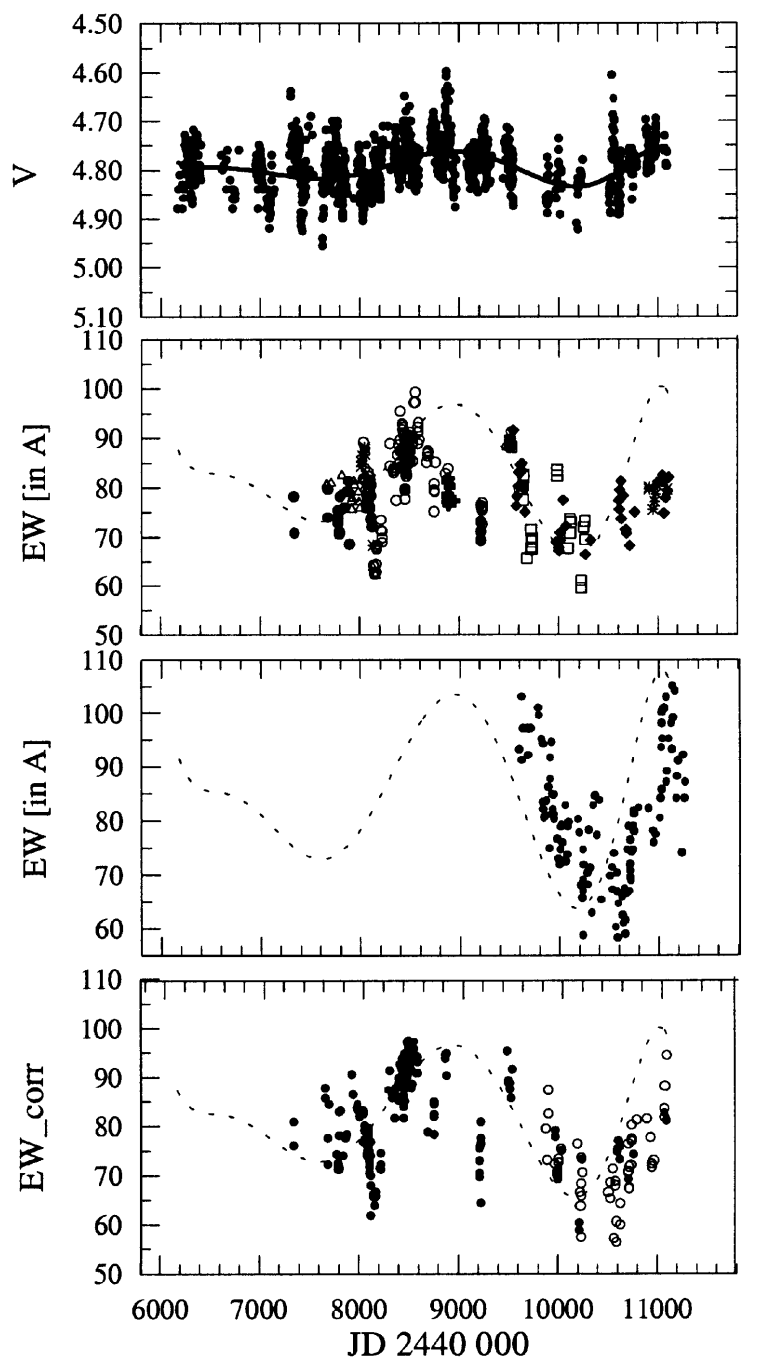

Fig. 3. Comparison between $V$-band variations (top panel) and changes in the $\mathrm{H} \alpha$ equivalent width uncorrected (middle panels) and corrected (bottom panel) for the effect of the changing continuum. The solid and the dashed lines represent the pattern of the SD variability obtained as a seventh-order polynomial fit to the $V$-band data. The purpose of the dashed lines is to guide the eye, emphasising the existence of a positive correlation between variations in $\mathrm{H} \alpha$ and changes in the stellar brightness through the SD phase.

apparent that, although some correspondence between the $V$-band variability through the 7.4-year SD-phase and the VLT spectral variability does exist, the two phenomena do not seem to be directly correlated.

However, the $\mathrm{H} \alpha$ line flux, which can be obtained from the equivalent width by removing the continuum normalisation, may be more physically meaningful than the equivalent width. For the $E W$ observations that were close enough in time to photometric observations to be considered simultaneous, we scaled the $E W$ s to a constant continuum level chosen to correspond to $V_{0}=4.8 \mathrm{mag}$. Thus, the measured $E W$ s were multiplied/divided by a factor $K$, where $\log K=0.4 \Delta V$ and $\Delta V=V(t)-V_{0}$ correspond- ingly when P Cygni is brighter/fainter. Strictly speaking, differences in the continuum flux at $\lambda=6563 \AA$ should be used, but in our case $\Delta V$ is a good approximation since the colour indices of P Cygni do not vary greatly (Fig. 1). The corrected $E W$ s from all observers, $W_{\text {corr }}$, are shown in the bottom panel of Fig. 3. Unfortunately, the lack of complete simultaneity between the spectroscopic and photometric observations has led to a noticeable reduction in the number of available $E W$ estimates. Nevertheless, one can see that the pattern of the VLT variability of $\mathrm{H} \alpha$ does not change significantly when the effect of the changing continuum is taken into account. In addition, it appears (if it is not an artefact of bad data sampling) that the corrected $E W$ data follow more closely the pattern of the SD variability. In summary, we conclude that: first, the VLT variability of $\mathrm{H} \alpha$ is intrinsic to the wind, not the photosphere; and, second, this variability is linked to the 7.4-year SD-phase.

A rough estimate of the relative variation in $\dot{M}$ that could result in the variations observed in $\mathrm{H} \alpha$ during the VLT variability can be derived by means of the scaling relation found by Puls et al. (1996). This relation connects mass-loss rate, terminal velocity, stellar parameters and $\mathrm{H} \alpha$ equivalent width and in the optically thick limit can be approximated as :

$\dot{M}($ thick $) \sim v_{\infty}^{3 / 4} R_{\star}^{3 / 2}\left[W_{\lambda}^{\prime \prime}\right]^{3 / 4}$

where $W_{\lambda}^{\prime \prime}=W_{\lambda}^{\text {obs }}+W_{\lambda, \text { phot }}^{\text {red }}$. In fact, this approximation was derived under the assumption of a $\beta$-velocity law with $\beta=1$ but it is appropriate for $\mathrm{P}$ Cygni as well since Puls et al. (1996) argued that at large $\dot{M}$ the value of $\beta$ is not of importance - for P Cygni $\beta=4$ (Pauldrach $\&$ Puls 1990). Thus, adopting $W_{\text {corr }}(\max )=95 \AA$ and $W_{\text {corr }}(\min )=75 \AA$ and neglecting the contribution of the photospheric absorption $\left(W_{\lambda, \text { phot }}^{\text {red }}=0\right)$ we derive, under the assumption of a constant stellar radius and wind terminal velocity, $\frac{\dot{M}(\max )}{\dot{M}(\min )}=1.19$. (Note that in P Cygni the contribution of the photospheric absorption to the total equivalent width of $\mathrm{H} \alpha$ is less than $2 \%$, i.e. negligible). This value is probably a bit overestimated since the wind terminal velocity of P Cygni is expected to change slightly and in opposite to the mass-loss rate (Markova et al. 2001). Thus we conclude that the observed increase of the $\mathrm{H} \alpha$ EW during the VLT variability could be associated with a $19 \%$ increase in the $\dot{M}$.

It seems that the LT variability of the $\mathrm{H} \alpha E W$ has a weak counterpart in the photometric behaviour of the star in view of a small depression in the light curve. Indeed, de Groot et al. (2001a,b) found, using Fourier technics, that photometric variations on a time scale of 500 days are certainly present. Colour information about this variability is not available. Based on the similarity of the time scale, we are tempted to identify this variation with the LT variability of $\mathrm{H} \alpha$. Thus we conclude that a positive correlation might exist between the $600^{\mathrm{d}}$ variability of $\mathrm{H} \alpha$ and the stellar brightness. 


\section{Discussion and conclusions}

We have studied the long-term spectral and photometric behaviour of P Cygni over an interval of about 14 years. Our survey is a natural continuation of the study of Markova et al. (2001), which we have supplemented with new data. We found that $\mathrm{P}$ Cygni has experienced a slow variation in apparent magnitude (amplitude of $\sim 0.1 \mathrm{mag}$, time scale of $\sim 7.4$ years) with a colour that is redder when the star brightens and vice versa. The variation showed a possible maximum in the winter of 1985 (between JD 2446000 and JD 2446200 ), a minimum in the winter of 1989 (between JD 2447500 and JD 2447700), a maximum in the fall of 1992 (between JD 2448800 and JD 2449000 ), and a minimum in the spring of 1996 (between JD 2450100 and JD 2450300 ). The amplitude and the time scale of this variation are typical of the so-called Short SD-phases observed in weak active S Dor variables, confirming the suggestion by van Genderen (2001).

Our simultaneous spectroscopic observations show that the equivalent width of $\mathrm{H} \alpha$ exhibits very long-term (VLT, time scale of $\sim 7$ years) variability with an amplitude of about $20 \AA$. It persists when equivalent width is converted to line flux by a correction for the effect of the changing continuum. Most of the time the VLT spectral variability follows the photometric variability through the 7.4-year SD-phase. In addition, we found that the LT variation in the $\mathrm{H} \alpha E W$ that was noted by Markova et al. (2001) (time scale $600^{\mathrm{d}}$, amplitude $\sim 30 \AA$ ) is probably recurrent. A positive correlation between this variation and changes in the $V$-band seems to exist. In summary, we conclude that the $\mathrm{H} \alpha E W$ experiences a $\sim 7$-year oscillation, which is positively correlated with the stellar brightness, and a $\sim 600^{\mathrm{d}}$ oscillation, which may be correlated with the stellar brightness, with the latter superimposed on the former. Using the scaling relation for $\mathrm{H} \alpha$ equivalent width (optically thick case) derived by Puls et al. (1996) for O-type stars we furthermore found that the observed increase in the $\mathrm{H} \alpha E W$ during the VLT variability can be associated with a $19 \%$ increase in the mass-loss rate. This result allows us to suggest that in $\mathrm{P}$ Cygni an increase in the stellar brightness, during the 7.4-year SD phase, is likely accompanied by increase in the mass-loss rate. In this behaviour P Cygni is similar to S Dor and R71.

De Groot et al. (2001b) report a Fourier analysis of $V$-band observations of $\mathrm{P}$ Cygni covering the interval from 1982 to 2000 and showing a possible 4.5-year cyclic oscillation in the stellar brightness which they recognised as a short SD-phase. Yet, the scatter around the fitted sine curve suggests that this cycle is not quite unambiguous. They conclude that it is not impossible that the observations after 1992 represent a cycle nearly twice as long. This supports our study. Thus, we conclude that, during the last two decades, although in quiescence, $\mathrm{P}$ Cygni experienced at least two low-amplitude SD cycles with several short-term microvariations (de Groot et al. 2001a,b; Markova 2000; Markova et al. 2001) superimposed.
Neither the mechanism causing the SD-phases, nor the region where this mechanism originates is known. Concerning the location of the origin of variability, two ideas have been announced. The first is that the SD-phases are an atmospheric phenomenon in which a drastic increase in mass-loss rate leads to the formation of a pseudophotosphere. In the second hypothesis, the variations are, at least partly, due to variations in the underlying stellar radius, i.e. they have a subphotospheric origin (Leitherer et al. 1988). De Koter et al. (1996), showed that typical S Dor variations of $\Delta V \sim 1$ to 2 mag cannot be due to formation of a pseudo-photosphere. On the other hand, those authors calculated that mass loss and velocity structure variations may in principle cause $V$-band variations of $\Delta V \simeq 0.2 \mathrm{mag}$ if the optical depth of the wind, $\tau_{\nu}$ (at $5555 \AA$ ), is initially of order unity. To estimate this quantity, they provided the formula:

$\tau_{\nu}=7.1 \times 10^{22} b_{3} \frac{\exp (17536 / T)}{T^{2 / 3}} \frac{\dot{M}^{2}}{v_{\infty}{ }^{2} R_{\mathrm{in}}{ }^{3} x_{\mathrm{o}}{ }^{3}} \Upsilon$.

In this expression, $\dot{M}$ is the mass-loss rate expressed in $M_{\odot} \mathrm{yr}^{-1}, R_{\text {in }}$ is the inner boundary radius in $R_{\odot}, v_{\infty}$ is the terminal flow velocity in $\mathrm{km} \mathrm{s}^{-1}, b_{3}$ is the nonLTE departure coefficient of the third level of hydrogen, and $x_{\mathrm{o}}=r_{\mathrm{o}} / R_{\text {in }}$ where $r_{\mathrm{o}}$ is a parameter determined such that both the velocity $v\left(r_{\mathrm{o}}\right)$ and the acceleration, $\mathrm{d} v /\left.\mathrm{d} r\right|_{r_{\mathrm{o}}}$, are continuous functions. $\Upsilon\left(a / v_{\infty}\right)$ is an integral that has several analytical solutions for specific values of $\beta$. Adopting $\dot{M} \simeq 1.9 \times 10^{-} 5 M_{\odot} \mathrm{yr}^{-1}$ (Scuderi et al. 1994; Markova et al. 2001), $R_{\text {in }}=R_{\star}=76 R_{\odot}$ (Pauldrach $\&$ Puls 1990), $T_{\text {wind }}=15000 \mathrm{~K}, v_{\infty}=230 \mathrm{~km} \mathrm{~s}^{-1}$ (Stahl et al. 1991; Markova \& de Groot 1997), $\beta=4$ (Pauldrach \& Puls 1990), $\Upsilon=0.30$ and $x_{\mathrm{o}}=x_{\mathrm{s}}=1.5$, we evaluated Eq. (1) to obtain $\tau_{\nu}=2.30$. This result indicates that the wind of $\mathrm{P}$ Cygni is probably sufficiently optically thick for the star to have a permanent pseudo-photosphere and hence, changes in visual magnitude of the order of 0.2 mag could be caused by mass-loss rate variations. Using Eq. (1) we furthermore estimated that a $19 \%$ increase in the mass-loss rate, derived in the present study, will move this pseudo-photosphere from 4.61 to $4.91 R_{\star}$, that is, will cause $\sim 7 \%$ increase in the apparent stellar radius, in good agreement with the result from the $U B V$ photometry (Markova et al. 2001). In summary, we conclude that the 7.4-year SD-phase established in P Cygni could be of atmospheric origin.

However, since P Cygni is close to its Atmospheric Edington Limit (AEL), one could expect that an increase in $R_{\star}$ and a decrease in $T_{\text {eff }}$ (due to some unknown reason) at constant luminosity will result in an increase in $\dot{M}$ and a decrease in $v_{\infty}$ (Lamers 1997), which will lead in turn (because of the high wind opacity) to an increase/decrease in the apparent stellar radius/temperature, i.e. exactly what we have observed. (In fact we have no direct measurements of $v_{\infty}$ during the 7.4-year SD-phase however Markova et al. (2001) showed, in terms of a simplified spherically-symmetric wind model, that variations in the 
velocity field, in opposite to mass-loss rate variability, are to be expected). Thus we see that it is impossible to judge, in the case of a star with optically thick wind and "small" photometric variations, what the primary cause of the SD variability is. In our opinion it seems more likely, at least for P Cygni, that the 7.4-year SD oscillations are a mixture of an expanding radius/decreasing temperature and an expanding pseudo-photosphere. Detailed investigation of the behaviour of the stellar luminosity during the SDphase could be clarifying since it might provide evidence for real expansion of the underlying star.

Another way for a hot massive star to generate variations in the basic parameters of its wind is by crossing the bi-stability limit, which was theoretically discovered by Pauldrach \& Puls (1990) and observationally established by Lamers et al. (1995). P Cygni is near the cool side of the bi-stability jump. Pauldrach \& Puls (1990) proposed a "feed-back" mechanism, based on the bi-stability, which could provide cyclic variations in the mass-loss rate of $\mathrm{P}$ Cygni. However, the predicted amplitude of variation in $\dot{M}$ is much higher (about a factor 4 ) and the time scale is much shorter (a factor 43) than during the 7 years observed. Thus we conclude that the 7.4-year SD-phase could not be caused by crossing the bi-stability limit.

Van Genderen (2001) has shown that LBVs or SD variables can be subject to two different SD-phases: the one on a time scale of years $(t<10 \mathrm{yr})-$ short-SD phase, the other on a time scale of decades $(t \geq 20 \mathrm{yr})$ - long$\mathrm{SD}$ phase. If both are present simultaneously the shorter one is superimposed on the longer one (van Genderen et al. 1997b). No SD-phases were found with cycle lengths between 10-20 yr. Another important characteristic of the phenomenon is its multi-periodicity/-cyclicity (van Genderen et al. 1997a,b; Sterken et al. 1996,1997, 1998). As already mentioned, there is no instability mechanism known yet for the SD phases. The time scale of the $\mathrm{SD}$ oscillations is obviously too long to be explained in terms of pulsation instability like strange-mode oscillations (Kiriakidis et al. 1993; Glatzel et al. 1999), gravitymode oscillations or relaxation oscillations (Stothers \& Chin 1995, 1996). On the other hand, the only mechanism that could produce phases of enhanced mass-loss rate in LBVs which repeat on time scale of years to decades are quasi-secular oscillations of the stellar envelope between dynamical stable and unstable states, according to the theoretical evolutionary tracks of Stothers \& Chin (1995, 1996, 2000). Van Genderen (2001) suggested that the long SD-phases could perhaps be identified with the "secular cycles" in Stothers \& Chin's models. However, these models do not predict any noticeable shift of the star on the HR diagram and the observed variations in the stellar brightness and colour should be therefore attributed to changes in the opacity of the wind. This fact limits the implication of the models only to LBV members that show increased mass-loss rate during SD-phases. Besides, the models do not predict a sharp physical division between S- and L-SD phases.
Concerning the LT $(\sim 600 \mathrm{~d})$ variation in the $\mathrm{H} \alpha E W$ curve it is perhaps interesting to note that Markova et al. (2001) have found, in terms of a simplified sphericallysymmetric, isothermal wind model, that this variation can be associated with changes in the optical depth of the wind due to a $26 \%$ alteration of the mass-loss rate, accompanied by a $\sim 28 \%$ variation in the velocity field (in opposite to each other). However, Markova (2000) argued, based on a detailed study of line-profile variability of a large sample of lines in the optical, that the LT variability is localised in only the outer part of the wind $\left(V \leq 0.82 V_{\infty}\right)$. So it seems likely that the LT variability is rather due to a large-scale time-dependent structure in the wind, than to variations in the mass-loss rate. At present, it is not clear whether the LT variability is linked to the appearance of Discrete Absorption Component or not.

Acknowledgements. NM is grateful to Henny Lamers for his valuable comments and suggestions. The constructive remarks of the referee, Dr. Arnout van Genderen, have helped to significantly improve the paper and are very much acknowledged. Support for observational research at Ritter Observatory is provided by the University of Toledo and by grants from the Fund for Astrophysical Research and from the American Astronomical Society Small Grants Program. Technical support at Ritter Observatory is provided by R. J. Burmeister. This work was supported by NSF to MES (Bulgaria) trough grant F-813/98 (N.M.).

\section{References}

Barlow, M. J., Drew, J. E., Meaburn, J., \& Massey, R. M. 1994, MNRAS, 268, L29

van Genderen, A. M. 2001, A\&A, 366, 508

van Genderen, A. M., de Groot, M., \& Sterken, C. 1997a, A\&AS, 124, 517

van Genderen, A., Sterken, C., \& de Groot, M. 1997b, A\&A, 318,81

Genet, R. M., Boyd, L. J., Kissell, K. E., et al. 1987, PASP, 99,660

Glatzel, M., Kiriakidis, M., Chernigovskij, S., \& Fricke, K. J. 1999, MNRAS, 303, 116

de Groot, M., \& Sterken, C. 2001, Proceedings Workshop P Cygni 2000: 400 years of progress, ASP Conf. Ser., (??), 288 de Groot, M., Sterken, C., \& van Genderen, A. M. 2001a, in P Cygni 2000: 400 years of progress, ed. M. de Groot, \& C. Sterken ASP Conf. Ser., in press

de Groot, M., Sterken, C., \& van Genderen, A. M. 2001b, A\&A, in prep.

Humphreys, R., \& Davidson, K. 1994, PASP, 106, 1025

Johnson, D. R. H., Barlow, M. J., Drew, J. E., \& Brinks, E. 1992, MNRAS, 255, 261

Kiriakidis, M., Fricke, K. J., \& Glatzel, W. 1993, MNRAS, 264, 50

de Koter, A., Lamers, H. J. G. L. M., \& Schmutz, W. 1996, A\&A, 306, 501

Lamers, H. J. G. L. M. 1997, ASP Conf. Ser., 120, 76

Lamers, H. J. G. L. M., Snow, T. P., \& Lindholm, D. M. 1995, ApJ, 455, 269

Langer, N., Hamann, W. R., Lennon, M., et al. 1994, A\&A, 290, 819 
Leedjärv, L. 1998, A\&A, 338, 139

Leitherer, C., Schmutz, W., \& Abbott, D. C., et al. 1989, ApJ, 346, 919

Markova, N. 2000, A\&AS, 144, 391

Markova, N. 2001, in P Cygni 2000: 400 years of progress, ed. M. de Groot, \& C. Sterken ASP Conf. Ser., in press

Markova, N., \& de Groot, M. 1997, A\&A, 326, 1111

Markova, N., Scuderi, S., de Groot, M., Markov, H., \& Panagia, N. 2001, A\&A, 366, 935

Meaburn, J., Lopez, J. A., Barlow, M. J., \& Drew, J. E. 1996, MNRAS, 283, L69

Meynet, G., Maeder, A., Schaller, D., \& Charbonnel, C. 1994, A\&AS, 103, 97

Mulliss, C. L. 1996, M.S. Thesis, U. of Toledo

Pauldrach, A., \& Puls, J. 1990, A\&A, 237, 409

Percy, J. R., Napke, A. E., Richer, M. G., et al. 1988, A\&A, 191, 248

Percy, J. R., Attard, A., \& Szczesny, M. 1996, A\&AS, 117, 255

Pollmann, E. 1999, Be Star Newsletter 34

Puls, J., Kudritzki, R.-P., Herrero, A., et al. 1996, A\&A, 305, 171
Schaller, G., Schaerer, D., Meynet, G., \& Maeder, A. 1992, A\&AS, 96, 269

Scuderi, S., Bonanno, G., Spadaro, D., et al. 1994, ApJ, 437, 465

Scuderi, S., Panagia, N., Stanghellini, C., et al. 1998, A\&A, 332,251

Skinner, C. J., Exter, K. M., Barlow, M. J., et al. 1997, MNRAS, 288, L7

Stahl, O., Mendel, H., Szeifert, Th., et al. 1991, A\&A, 244, 467

Stahl, O., Kaufer, A., Wolf, B., et al. 1994, JAD vol. 1, dir 3

Sterken, C., de Groot, M., \& van Genderen, A. 1996, A\&AS, 116,9

Sterken, C., de Groot, M., \& van Genderen, A. 1997, A\&A, 326,640

Sterken, C., de Groot, M., \& van Genderen, A. 1998, A\&A, 333,565

Stothers, R. B., \& Chin, C. W. 1995, ApJ, 451, L60

Stothers, R. B., \& Chin, C. W. 1996, ApJ, 468, 842

Stothers, R. B., \& Chin, C. W. 2000, ApJ, 540, 1041

Taylor, M., Nordsieck, R. E., Schulte-Ladbeck, R. E., et al. 1991, AJ, 102, 1197 